\title{
Reconstruction of vertical profiles of Chlorophyll concentration
}

\author{
Roberto P. Souto*a ${ }^{* a}$ Milton Kampel ${ }^{\mathrm{b}}$, Haroldo F. de Campos Velho ${ }^{\mathrm{c}}$, Stephan Stephany ${ }^{\mathrm{c}}$ \\ ${ }^{a}$ Informatics Institute - Federal University of Rio Grande do Sul (II-UFRGS), Av. Bento Gonçalves, \\ 9500 - Porto Alegre, RS, Brazil 91501-970 \\ ${ }^{\mathrm{b}}$ Remote Sensing Division - DSR, ${ }^{\mathrm{c}}$ Laboratory for Computing and Applied Mathematics - LAC - \\ National Institute for Space Research - São José dos Campos, SP, Brazil 12227-010
}

\begin{abstract}
This work presents the multispectral reconstruction of the vertical profile of Chlorophyll concentration (Chl-a) in Case 1 waters. In such ocean waters, optical properties are driven by the presence of phytoplankton, allowing the use of biooptical models, where the absorption and scattering coefficients are related with Chl-a. The vertical profile of Chl-a is reconstructed from experimental measurements of water-leaving radiances $(\mathrm{nLw})$ at 10 wavelengths. These radiances are considered for a discrete number of upward polar directions. The inverse problem is formulated as an optimization problem, and iteratively solved by the ACO using the radiative transfer equation as direct model. The objective function is given by the square difference between computed and experimental radiances. For each iteration a population of candidate solutions is generated, pre-selected and evaluated by means of the objective function. Each candidate solution corresponds to a discrete Chl-a profile. The radiative transfer equation is solved for each candidate solution yielding the radiances that are used in the objective function to evaluate it. Since this equation requires the absorption and scattering coefficients, these are calculated using bio-optical models. The radiative transfer equation is solved using the Laplace transform discrete ordinate $\mathrm{LTS}_{N}$ method. A parallel implementation of the ACO is employed and executed in a distributed memory machine. The multispectral approach allows estimating the vertical profile using only nLw, instead of in situ measurements on several depths.
\end{abstract}

Keywords: optical properties identification, Case-1 water, radiative transfer, inverse problems, ant colony optimization.

\section{INTRODUCTION}

In the last decades, the development of inversion methodologies for radiative transfer problems has been an important research topic in many branches of science and engineering [1,2]. The direct or forward radiative transfer problem in hydrologic optics, in the steady state, involves the determination of the radiance distribution in a body of water, given the boundary conditions, source term, inherent optical properties (IOPs), as the absorption and scattering coefficients, and the phase function. The inverse radiative transfer problem arises when physical properties, internal light sources and/or boundary conditions must be estimated from radiometric measurements of the underwater light field. A challenge in the inverse hydrologic optics problem is to determine the IOPs, only considering water-leaving radiance.

The inverse problem is formulated as an optimization problem and iteratively solved using a recent intrinsic regularization scheme $[3,4]$ coupled to a standard Ant Colony Optimization (ACO). The regularization scheme preselects candidate solutions based on their smoothness, quantified by a Tikhonov norm as proposed by Preto et al. [3] in a former work.

An objective function is given by the square difference between computed and experimental radiances at every iteration. Each candidate solution corresponds to a discrete Chlorophyll concentration profile (Chl-a).

In this work, the Chl-a profile is reconstructed from multi-spectral water-leaving radiances of ocean surface, following the methodology presented by Chalhoub and Campos Velho [5]. Vertical values of the absorption and scattering coefficients are estimated from the Chl-a profile by means of bio-optical models [6], and after they are employed in the radiative transfer model at each inverse iteration.

Coastal Ocean Remote Sensing, edited by Robert J. Frouin, ZhongPing Lee, Proc. of SPIE Vol. 6680, 66800B, (2007) · 0277-786X/07/\$18 · doi: 10.1117/12.738116 


\section{RADIATIVE TRANSFER MODEL IN NATURAL WATERS}

The radiative transfer equation (RTE) models the transport of photons through a medium [7]. Light intensity is given by a directional quantity, the radiance $L$, that measures the rate of energy being transported at a given point and in a given direction. For a given horizontal plane, this direction is defined by a polar angle $\theta$ (relative to the normal of the plane) and a azimuthal angle $\varphi$ (a possible direction in that plane). At any point of the medium, light can be absorbed, scattered or transmitted, according to the absorption $(a)$ and scattering $(b)$ coefficients and to a scattering phase function that models how light is scattered in any direction. An attenuation coefficient $c$ is defined as $c=a+b$ and the geometrical depth is mapped to a optical depth $\underline{\tau}$ that imbeds $c$. Assuming a plane-parallel geometry, and making the radiance $L_{\lambda}(\tau, \mu, \varphi)=L_{\lambda}$, the one dimensional integro-differential RTE, can be written as:

$$
\mu \frac{d}{d \tau} L_{\lambda}+L_{\lambda}=\frac{\varpi_{0}(\tau, \lambda)}{4 \pi} \int_{0}^{2 \pi} \int_{-1}^{1} p\left(\mu, \varphi \rightarrow \mu^{\prime}, \varphi^{\prime}\right) L_{\lambda} d \mu^{\prime} d \varphi^{\prime}+S_{\lambda}(\tau)
$$

subjected to boundary conditions

$$
L_{\lambda}(0, \mu, \varphi)=F \delta\left(\mu-\mu_{0}\right)\left(\varphi-\varphi_{0}\right)
$$

and

$$
L_{\lambda}(\zeta,-\mu, \varphi)=0
$$

where $\mu \in[-1,1]$ and $\varphi \in[0,2 \pi]$ are respectively the cosine of the polar angle $\theta$ and the azimuthal angle, $\varpi_{0}(\tau, \lambda)=b(\tau, \lambda) / c(\tau, \lambda)$ is the single scattering albedo, and the scattering phase function $p\left(\mu, \varphi \rightarrow \mu^{\prime}, \varphi^{\prime}\right)$, gives the beam angular distribution, mapping the beam direction $(\mu, \varphi)$ to the scattered direction $\left(\mu^{\prime}, \varphi^{\prime}\right)$ in the medium. The source term is $S$.

In Equation (2), $\mu_{0}$ and $\varphi_{0}$ are respectively the cosine of the incident polar and azimuthal angles, and $F$ express the net flux of radiation integrated for all directions, i.e., the irradiance.

Equation (1) can be rewritten for the case of azimuthal symmetry (no dependence on $\mathrm{j}$ ), isotropic medium (isotropic phase function $\left.p\left(\mu, \varphi \rightarrow \mu^{\prime}, \varphi^{\prime}\right)=1\right)$ and absence of source term, as follows

$$
\mu \frac{d}{d \tau} L_{\lambda}(\tau, \mu)+L_{\lambda}(\tau, \mu)=\frac{\varpi_{0}(\tau, \lambda)}{2} \int_{-1}^{1} L_{\lambda}(\tau, \mu) d \mu^{\prime}
$$

subjected to boundary conditions

$$
L_{\lambda}(0, \mu)=F \delta\left(\mu-\mu_{0}\right)
$$

and

$$
L_{\lambda}(\zeta,-\mu)=0
$$

A heterogeneous medium, as shown in Figure 1, can be modeled as a set of $R$ homogeneous finite layers.Optical variable $\underline{\tau}$ is discretized in $\underline{R+1}$ values, varying from $\underline{\tau}_{0}=\underline{0}$ up to $\underline{\tau}_{\underline{R}}=\zeta$, where $\zeta$ is the medium optical depth. Then, for $\underline{r}=1,2, \ldots, R$ and $\underline{\mu} \in \underline{(0,1]}$, the problem in this multi-region geometry can be given by

$$
\mu \frac{d}{d \tau} L_{r, \lambda}(\tau, \mu)+L_{r, \lambda}(\tau, \mu)=\frac{\varpi_{r}(\lambda)}{2} \int_{-1}^{1} L_{r, \lambda}(\tau, \mu) d \mu^{\prime}
$$

with 


$$
\varpi_{0}(\tau, \lambda)=\varpi_{r}(\lambda)=\frac{b_{r}(\lambda)}{c_{r}(\lambda)}=\frac{b_{r}(\lambda)}{a_{r}(\lambda)+b_{r}(\lambda)}
$$

constant in the region $r$, for any value of $\underline{\tau}$, where $\underline{c}_{r}(\lambda), \underline{a}_{r}(\lambda)$, and $\underline{b}_{r}(\lambda)$ are respectively the attenuation, the absorption and the scattering coefficients, for a given wavelength $\lambda$.

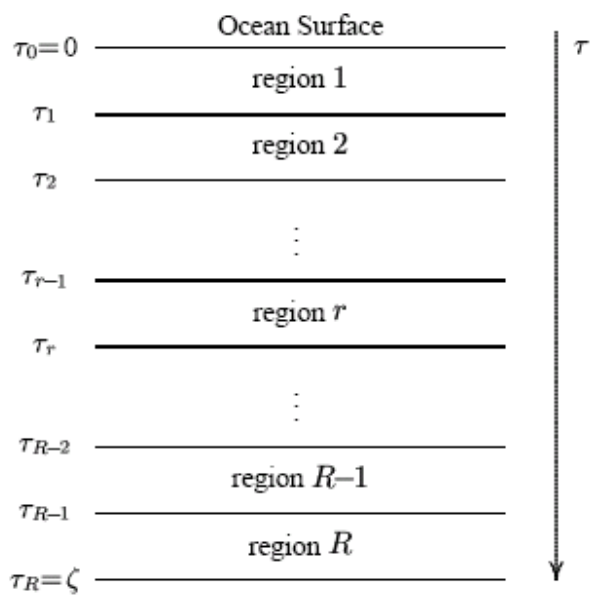

Fig. 1. Plane-parallel geometry with $R$ regions that correspond to horizontal layers (adapted from Chalhoub and Campos Velho [5]).

Equation (5) is subject to boundary conditions

$$
L_{1, \lambda}\left(\tau_{0}, \mu\right)=L_{R, \lambda}\left(\tau_{R},-\mu\right)=0
$$

and for the region interfaces in $\underline{r}=1,2, \ldots, R-1$, is assumed the continuity of the radiance.

$$
L_{r, \lambda}\left(\tau_{r}, \pm \mu\right)=L_{r+1, \lambda}\left(\tau_{r}, \pm \mu\right)
$$

for $\mu \in(0,1]$.

In the discrete ordinate method [8] the Equation (5) is approximated by a collocation method, where the $\mu$ integral is computed by the $N_{\mu}$-order Gauss-Legendre quadrature formula, with nodes $\left\{\mu_{i}\right\}$ and weights $\left\{\eta_{i}\right\}$, yielding a set of differential equations. The value of $\underline{\mu}$ is then discretized in $\underline{\mu}_{i}$, with $j=1,2, \ldots, N_{\mu}=2 n$ (even number), that are the discrete ordinate directions. The radiative transfer equation is then expressed as the related discrete ordinate equations, also known as $\underline{S}_{\underline{N}}$ equations:

$$
\mu_{j} \frac{d}{d \tau} L_{r, \lambda}\left(\tau, \mu_{j}\right)+L_{r, \lambda}\left(\tau, \mu_{j}\right)=\frac{\varpi_{r}(\lambda)}{2} \sum_{i=1}^{N_{\mu}} \eta_{i} L_{r, \lambda}\left(\tau, \mu_{i}\right)
$$

and the boundary conditions are

$$
L\left(\tau_{0}, \mu_{j}\right)=0, \quad j=1,2, \ldots, N_{\mu} / 2
$$

and 


$$
L\left(\tau_{R}, \mu_{j}\right)=0, \quad j=n+1, n+1, \ldots, N_{\mu}
$$

The discretized RTE is solved in this work by the $\mathrm{LTS}_{N}$ method [9], that generates a Optimization of equations of $\operatorname{order}(R+1) \times N_{\mu}$.

\subsection{Chlorophyll and IOPs relationship}

Bio-optical models are employed in this work to correlate the absorption and scattering coefficients to the Chlorophyll concentration. These coefficients are assumed to be constant in each region. Therefore discrete values $a_{r}$ and $b_{r}$ can be estimated for each region from the discrete values $C_{r}$.

Usually, Chlorophyll profiles can be represented according Gaussian distributions [6]. A particular profile, corresponding to the Celtic Sea was considered:

$$
C(z)=0.2+\frac{144}{9 \sqrt{2 \pi}} \exp \left[-\frac{1}{2}\left(\frac{z-17}{9}\right)^{2}\right]
$$

where $z$ is the depth in meters and $C(z)$ is given in $\mathrm{mg} / \mathrm{m}^{3}$. This profile can be seen in results section of this work. A biooptical model was formulated by Morel [10] for the absorption coefficient,

$$
a_{r}(\lambda)=\left[a^{w}+0.06 a^{c} C_{r}^{0.65}\right]\left[1+0.2 e^{-0.014(\lambda-440)}\right]
$$

where $a^{w}$ is the pure water absorption and $a^{c}$ is a nondimensional, statistically derived Chlorophyll specific absorption coefficient, and $\lambda$ is the considered wavelength, while another Chlorophyll correlation was formulated by Gordon and Morel [11] for the scattering coefficient,

$$
b_{r}(\lambda)=\left(\frac{550}{\lambda}\right) 0.30 C_{r}^{0.62}
$$

The considered Celtic Sea profile refers to a type of water that presents a high concentration of phytoplankton in comparison to organic particles [12]. The values of $a^{w}$ and $a^{c}$ depend on the wavelength and can be found in tables [6].

\section{ANT COLONY OPTIMIZATION}

The Ant Colony Optimization (ACO) is a method that employs a metaheuristic based on the collective behaviour of ants chosing a path between the nest and the food source [13]. Each ant marks its path with an amount of pheromone and the marked path is further employed by other ants as a reference. As an example of this, the sequence in Figure 2 shows how ants, trying to go from point A to point E (I), behave when an obstacle is put in the middle of the original path, blocking the flow of the ants between points B and D (II). Two new paths are then possible, either going to the left of the obstacle (point $\mathrm{H}$ ) or to the right (point $\mathrm{C}$ ). The shortest path causes a greater amount of pheromone to be deposited by the preceding ants and thus more and more ants choose this path (III).

In the ACO optimization method, several generations of ants are produced. For each generation, a fixed amount of ants $(n a)$ is evaluated. Each ant is associated to a feasible path and this path represents a candidate solution, being composed of a particular set of edges of the graph that contains all possible solutions. Each ant is generated by choosing these edges on a probabilistic basis.

This approach was succesfully used for the Traveling Salesman Problem (TSP) and other graph-like problems [13]. The best ant of each generation is then chosen and it is allowed to mark with pheromone its path. This will influence the creation of ants in the further generations. The pheromone put by the ants decays due to an evaporation rate. Finally, at the end of all generations, the best solution is assumed to be achieved. 


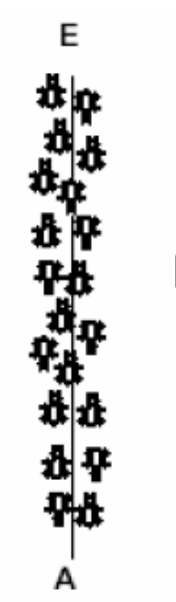

(I)

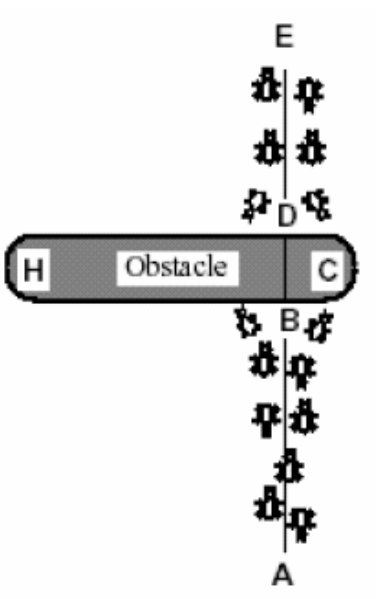

(II)

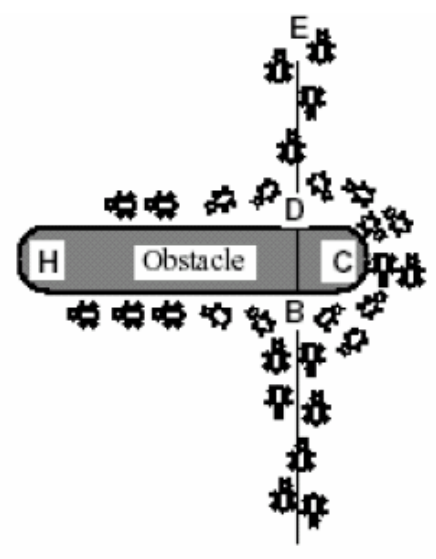

A

(III)

Fig. 2- Ants overcoming an obstacle in the trail (from Ref. [13]).

A solution is composed of linking $n s$ nodes and in order to connect each pair of nodes, $n p$ discrete values can be chosen. This approach was used to deal with a continuous domain. Therefore, there are $n s \times n p$ possible paths $[\mathrm{i}, \mathrm{j}]$ available. Denoting by $\rho$ the pheromone decay rate, the amount of pheromone $T_{j j}$ at generation $\mathrm{t}$ is given by:

$$
T_{i j}(t)=(1-\rho) T_{i j}(t-1) \quad t=1,2, \ldots, \text { mit }
$$

where mit is the maximum number of iterations. The initial amount of pheromone, at $t=0$, is calculated as sugested in [14] using an evalutation $Q$ of the function to be optimized obtained with a greedy heuristics:

$$
T_{i j}(0)=T_{0}=\frac{1}{n s \cdot Q}
$$

The probability of a given path $[i, j]$ be choosed at each ants generation, is then

$$
P_{i j}(t)=\frac{\left[T_{i j}(t)\right]^{\alpha}\left[\eta_{i j}(t)\right]^{\beta}}{\sum_{l}\left\{\left[T_{i l}(t)\right]^{\alpha}\left[\eta_{i l}(t)\right]^{\beta}\right\}}
$$

where $l \in[1, n p]$ and $\eta_{i j}$ is the visibility/cost of each path, a concept that arises from the TSP, where the cost is the inverse of the distance of a particular path. The parameters $\alpha$ and $\beta$ are weights that establish the trade-off between the influence of the pheromone and the visibility in the probability of each path. In this work, whe are using $\alpha=1.0$ and $\beta=0.0$, since this distance-dependent visibility approach is not applicable in the Chlorophyll profile estimation inverse problem.

Moreover, there is a further scheme for the choice of a path for a new ant. According to a roulette, a random number in the range $[0,1]$ is generated for the new ant and it is compared with a parameter $q_{0}$ chosen for the problem. If the random number is greater than this parameter, the path is taken according to $P_{i j}$. If not, the most marked path is assigned.

A parallel implementation of the Ant Colony Optimization [15,16] was executed in a distributed memory machine Parallelization is important since this problem is very computationally intensive. 


\section{INVERSION SCHEME}

The inverse problem is formulated according to an implicit approach, leading to an optimization problem [17]. The algorithm is expressed as a constrained nonlinear optimization problem, in which the direct problem is iteratively solved for successive approximations of the unknown parameters. Iteration proceeds until an objective-function, representing the least-square fit of model results and experimental data added to a regularization term, converges to a specified small value.

The corresponding inverse radiative transfer problem arises when physical properties, internal light sources and/or boundary conditions must be estimated from radiometric measurements of the underwater light field.

The set of parameters to be estimated is $R+1$ discrete values of the Chlorophyll concentration $C_{r}$, for $r=0,1, \ldots, R$ at optical depths $\tau_{r}$ taken at the upper interface of the regions. Experimental data are the discrete radiances $L^{\exp }\left(\tau_{0}, \mu_{i}, \lambda_{j}\right)$ for $i=1,2, \ldots, N_{\mu} / 2$ and $j=1,2, \ldots, N_{\lambda}$. Therefore, the $R+1$ discrete values of the concentration are estimated from $N_{\mu} / 2 \times N_{\lambda}$ sprectral radiance values right above of sea surface. The objective function $J(\boldsymbol{C})$ is given by the square difference between experimental and model radiances plus a regularization term.

$$
J(\boldsymbol{C})=\sum_{i=1}^{N_{\mu} / 2} \sum_{j=1}^{N_{\lambda}}\left[L^{e x p}\left(\tau_{0},-\mu_{i}, \lambda_{j}\right)-L_{\boldsymbol{C}}\left(\tau_{0},-\mu_{i}, \lambda_{j}\right)\right]^{2}+\gamma \Gamma(\boldsymbol{C})
$$

where $\Gamma(\boldsymbol{C})$ is the regularization function, that is weighted by a regularization parameter $\gamma$. For instance, the 2 nd order Tikhonov regularization [18] is defined by

$$
\Gamma(\boldsymbol{C})=\sum_{r=0}^{R-2}\left(C_{r}-2 C_{r+1}+C_{r+2}\right)^{2}
$$

\subsection{Intrinsic regularization}

In the current work, a ACO-based inverse solver with an implicit regularization scheme $[3,19]$ is employed without the regularization term $(\gamma=0)$ showed in Equation (17). As a smooth profile is required, this is a known information about the inverse solution. Such knowledge is included in the generation of the candidate solutions by means of preselecting the smoother ants according to the 2nd order Tikhonov norm. Actually, a kind of pre-regularization is performed. Therefore the usual regularization term is not required.

\subsection{Concavity criterion}

Besides the smoothness, additional information is also used to compute the inverse solution: the concavity of the Chlorophyll profile that is verified by means of its second derivative. Since only curves with negative concavity are expected, a penalty is assigned to profiles with positive concavity. For each of these profiles, an overhead value is added to the evaluated objective function (Equation 17).

\section{RESULTS AND DISCUSSIONS}

The inverse solver was tested for a multi-region $(\underline{R=9})$ offshore ocean water radiative transfer problem with azimuthal symmetry, using multi-spectral noiseless radiance data. This data is related to the emerging radiances at the water surface and include $\underline{N}_{u} / 2=10$ polar directions for each of the $\underline{N}_{2}=10$ wavelengths. In the considered test cases, data was used to simulate the experimental values obtained with a profiler of natural fluorescence (PNF, Biospherical Instruments), during a cruiser in Brazilian southeast coast, in autumn of 2003 [20]. The first case is an unimodal profile, with one peak of Chlorophyll concentration and, as can be seen in Figure 3, it is similar to the Gaussian profile mentioned in Section 2.1.

In its turn, the second reconstructed profile showed in Figure 4 has not one, but two peaks of Chlorophyll concentration, and for this reason it is a bimodal profile. For both cases, are presented reconstruction results using the inverse problem formulation, solved by an ACO algorithm version that embeds the intrinsic regularization explained in 
Section 4.1. It is also made a comparison between results obtained with the second derivative criterion not activated and activated.

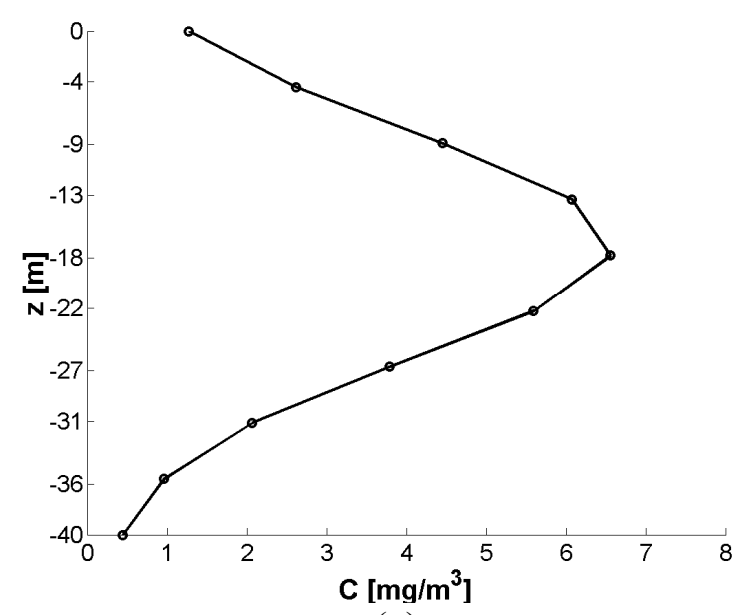

(a)

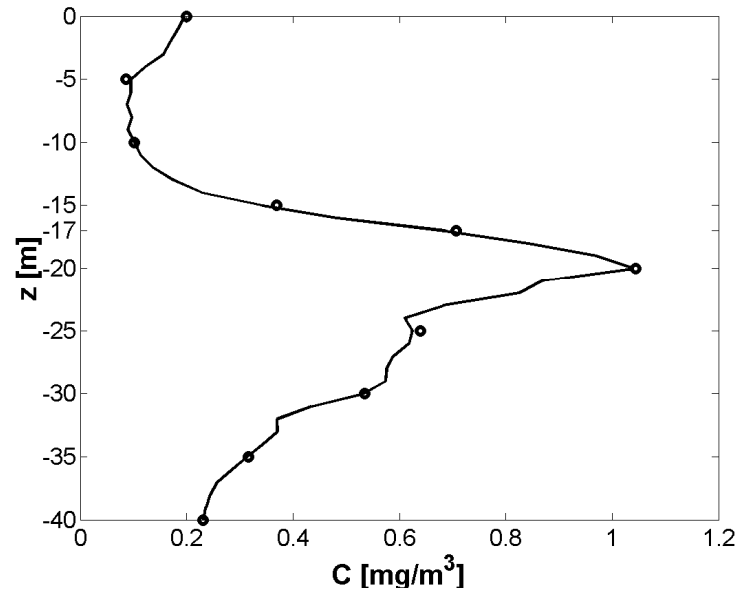

(b)

Fig. 3. Gaussian model and the measured unimodal Chlorophyll concentration profiles.

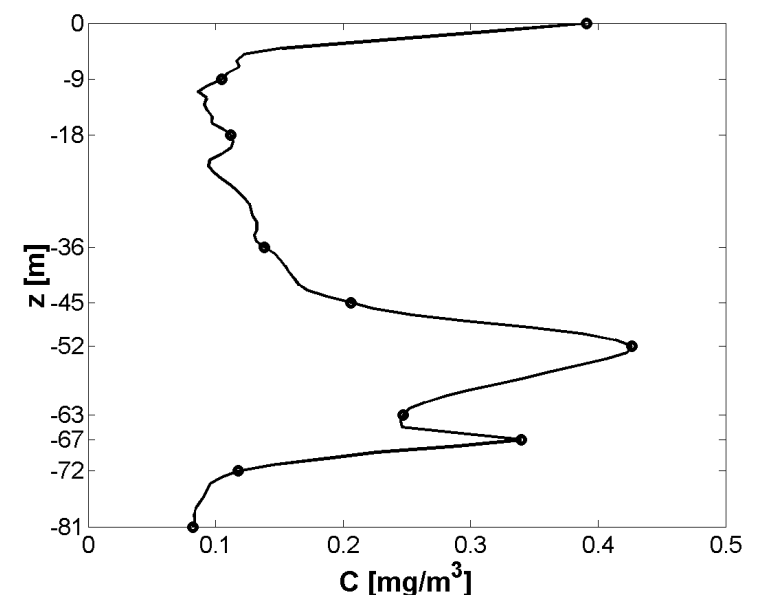

Fig. 4. Measured bimodal Chlorophyll concentration profile.

In previous work [21], it had already obtained very good results of Gaussian profile reconstruction using the same methodology employed here. Furthermore, the first attempt in order to reconstruct the measured bimodal profile was done in [22]. However, instead of using multispectral radiances obtained only in sea surface, it was used radiances in each depth of the Chlorophyll profile, available with just one wavelength though. In this situation, the reconstructions is simpler than the surface multispectral case, and were reached good results. Then, in this work the multispectral inverse problem formulation is tested in the reconstruction of a real measured chlorophyll profile, in place of Gaussian one.

\subsection{Reconstruction of the unimodal Chlorophyll profile}

The tuning of the parameters in the ACO may have a big influence in the results. The ACO implementation required adjustment of parameters as the pheromone decay rate $(\varrho)$, and $q_{0}$, used in the roulette scheme. There are other 
parameters in the process, such as the number of possible paths $(n p)$ between each pair of the $n s$ nodes, the number of ants $n a$, or the maximum number of iterations mit.

These values are shown in Table 1, and they as used for the test cases. The ACO was executed using $n a=300$ and pre selecting $1 / 12$ of these ants $\left(n a_{p}=25\right)$ according to their smoothness. The range of search for the Chlorophyll concentration varies from $0.0003(1.0 / 3000.0)$ up to $1.5(3000.0 / 2000.0)$. Different seeds are used in the ACO for the generation of pseudo-random numbers. It can be seen in the results that different seeds imply to different reconstructions.

Table 1. Seeds and ACO parameters.

\begin{tabular}{|c|ccccccc|}
\hline Seeds (10) & \multicolumn{6}{|c|}{$3,15,21,31,45,63,77,81,95,99$} \\
\hline ACO & $\mathrm{ns}$ & $\mathrm{np}$ & $\mathrm{na}$ & $\mathrm{na}_{\mathrm{p}}$ & mit & $\rho$ & $\mathrm{q}_{0}$ \\
\cline { 2 - 8 } parameters & 10 & 3000 & 300 & 25 & 400 & 0.03 & 0.0 \\
\hline
\end{tabular}

An intrinsic regularization was used, with $\gamma=0$, and the smoothness-based pre-selection of the ants was performed using the second order Tikhonov norm. Moreover, a new criterion was added to ACO algorithm: Chlorophyll profiles with negative concavity are preferred. Figure 5 shows the reconstruction of the Chlorophyll concentration profile, with the average profile obtained from the profiles that correspond to all the 10 seeds. It was employed ACO with only preselecting strategy (Figure 5a), and a retrieval using also the second derivative criterion from the 5 meters depth (Figure $5 b$ ), getting a better average solution, particularly near the middle of the profile.

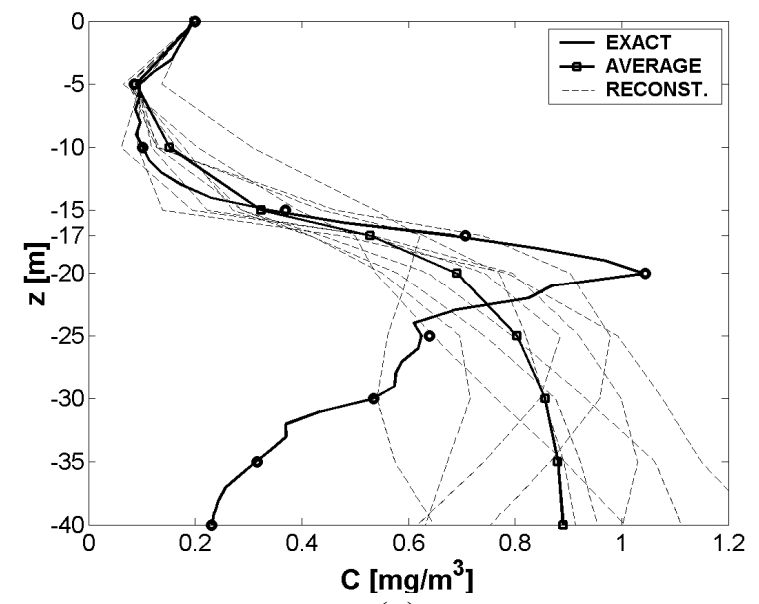

(a)

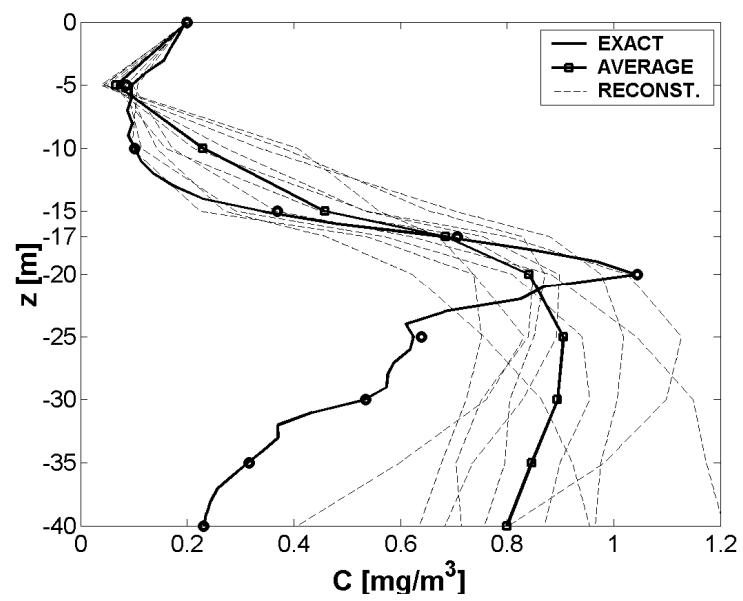

(b)

Fig. 5. Unimodal Estimated Chlorophyll average concentration profile (solid line):

(a) without $2^{\text {nd }}$ derivative criterion; (b) $2^{\text {nd }}$ derivative criterion activated.

In order to improve the inverse solution, it was used a strategy where is supposed the wanted profile has a peak in the $6^{\text {th }}$ depth, according analysis of profiles obtained with 10 seeds. After that, it is performed an average regarding to the top 5-Chlorophyll concentration peak profiles. A good estimation was obtained only for the upper part of the curve (average profile) with poorer agreement to the lower part of the profile (depth below to the peak - see Figure 6a).

From now on a further two-step strategy, previously used [21] in the Gaussian profile estimation, is adopted: step-1 the estimation has already performed for the whole profile, and then, in step-2, the reconstruction is carried out only for the lower part of the curve. In the step-2, each ant is still related to the whole profile, but the values obtained in the step-1 for the upper part of the water layer are frozen, obtaining the average profile shown in Figure $6 \mathrm{~b}$. A closer agreement with the exact solution was obtained using the two-steps scheme. 
The two-step scheme presented in the previous work [19], five profiles were employed to perform the average profile. Such profiles are shown in Figure $4 \mathrm{a}$ with greater negative concavity. The concavity criterion is an a posteriori inference procedure.

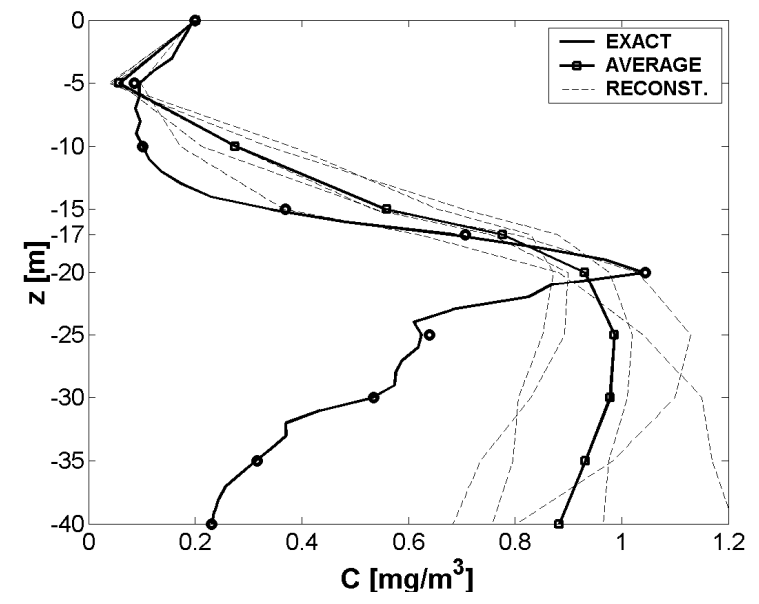

(a)

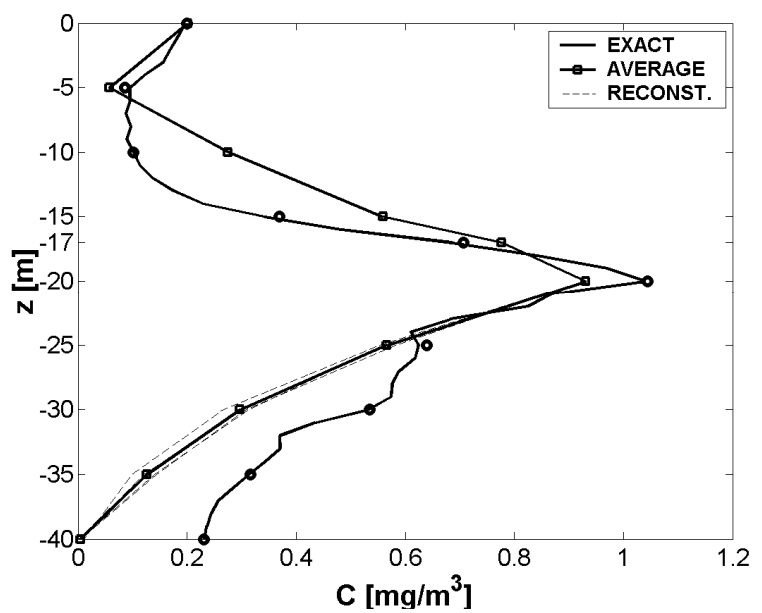

(b)

Fig. 6. Unimodal Chlorophyll concentration profiles identified for the 5 most peak ones:

(a) without $2^{\text {nd }}$ derivative criterion; (b) $2^{\text {nd }}$ derivative criterion activated.

\subsection{Reconstruction of the bimodal chlorophyll profile}

The ACO parameter values for this test case are the same already shown in Table 1 . The only difference is regarding to range of search for the Chlorophyll concentration. It now varies from $0.0003(1.0 / 3000.0)$ up to $0.5(3000.0 / 6000.0)$. In Figure 7 is shown the average Chlorophyll concentration profile, obtained using ACO with only pre-selecting strategy (Figure $7 \mathrm{a}$ ), and also with concavity criterion.

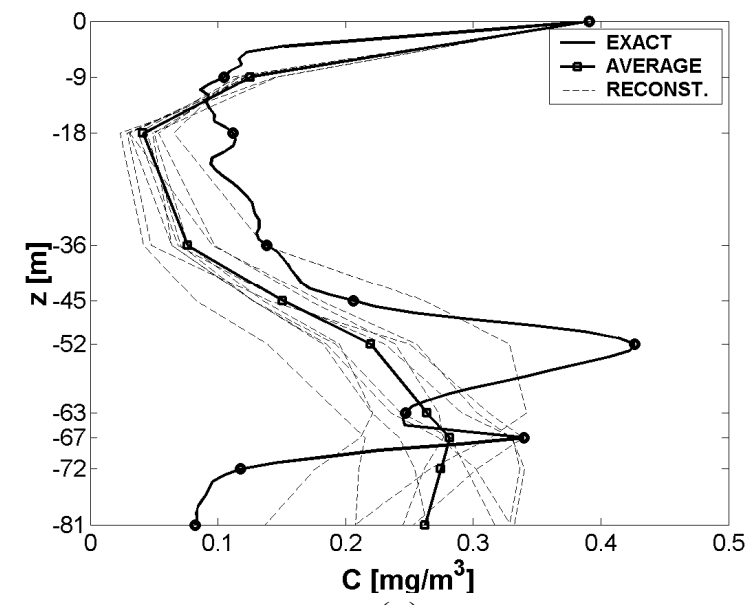

(a)

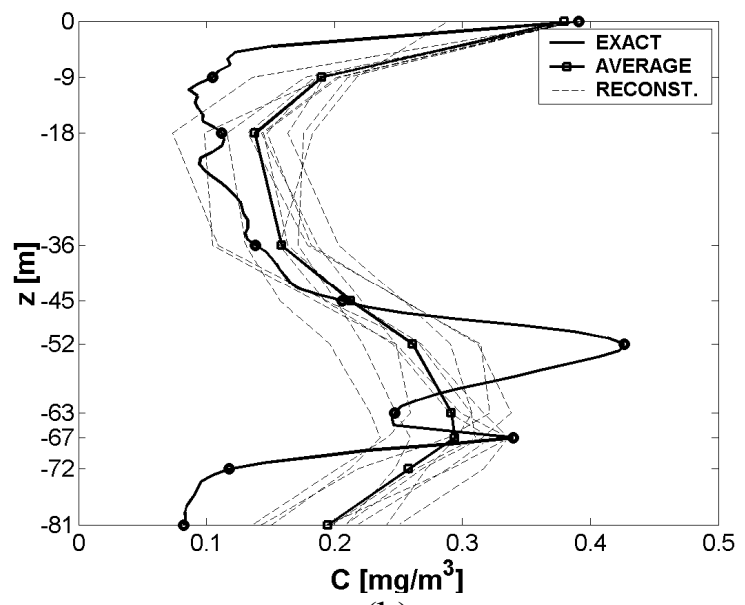

(b)

Figure 7: Bimodal Chlorophyll concentration profile identified:

(a) without $2^{\text {nd }}$ derivative criterion, (b) $2^{\text {nd }}$ derivative criterion activated. 
Although the exact bimodal profile does not fit to negative concavity as well as the unimodal profile, both seeds and average profiles obtained looks better than results reach with the later case. The average profile is already good enough, and was not necessary other strategy in order to improve it.

\section{CONCLUSIONS}

A multi-spectral reconstruction of Chlorophyll concentration profiles was successfully achieved by the use of an innovative application of the proposed inverse methodology. The absorption and scattering coefficients are computed from the Chlorophyll concentration profile using bio-optical models. The inverse solver employs a modified metaheuristics that combines a standard ACO algorithm and an intrinsic pre-regularization scheme. Significant improvement was reached with the use of the concavity criterion to compute the profiles.

The direct model is described by the radiative transfer equation, solved by the $\mathrm{LTS}_{N}$ method. The pre-regularization scheme was applied to the ACO algorithm in order to ensure smoothness in the generation/selection of the ants. This scheme was successfully used in a previous work [3], where usual regularization scheme - the objective function as given by Equation (17) - did not produce good results.

Another contribution in our inverse procedure is to use of a two-step reconstruction associated with the concavity criterion. However, this strategy was particularly effective for the unimodal profile case, since for the bimodal profile reconstruction, the use of negative concavity criterion was already enough. This result can indicate a more general applicability of this criterion in Chlorophyll concentration profile estimation, not only for unimodal ones.

In the test cases presented here, results show good agreement with the exact solution. In addition, the implemented parallel ACO code reduced the processing time, being the evaluations of the objective function distributed among processors. This is a relevant point in inverse problems, where thousands of iterations can typically be required.

Studies are underway to perform reconstructions using noisy data and more complex test cases: anisotropic scattering and/or the use of azimuthal-dependent data. Further steps may include reconstruction of multi-modal Chlorophyll concentration profiles, and test cases related to turbid waters (coastal oceans, harbor waters, and rivers).

\section{ACKNOWLEDGEMENTS}

Author R.P. Souto acknowledges Parallel and Distributed Processing Group (GPPD) at II-UFRGS, where all processing of ACO code was performed in 20-dual nodes cluster of this group.

\section{REFERENCES}

1. H.R. Gordon, Inverse methods in hydrologic optics. Oceanology, 44, 9 (2002).

2. N.J. McCormick Inverse radiative transfer problems: a review. Nuclear Science and Engineering, 112, 185 (1992).

3. A.J. Preto, H.F. de Campos Velho, J.C. Becceneri, N.N. Arai, R.P. Souto, S. Stephany, A new regularization technique for an ant-colony based inverse solver applied to a crystal growth problem, 13th Inverse Problem in Engineering Seminar, (IPES-2004), 14-15 June, University of Cincinnati, Ohio, USA, 147 (2004).A. Eisenberg, Guide to Technical Editing, Oxford University, New York, 1992.

4. R.P. Souto, H.F. de Campos Velho, S. Stephany, S. Sandri, Reconstruction of Chlorophyll Concentration Profile in Offshore Ocean Water using a Parallel Ant Colony Code, 16th European Conference on Artificial Intelligence (ECAI2004), Hybrid Metaheuristics (HM-2004), 22-24 August, Valencia, Spain, 19 (2004).

5. E.S. Chalhoub, H.F. de Campos Velho, Multispectral reconstruction of bioluminescence term in natural waters. Applied Numerical Mathematics, 47, 365 (2003).

6. C.D. Mobley, Light and Water: Radiative Transfer in Natural Waters, Academic Press, 1994, p. 592.

7. H.R. Gordon, Remote sensing marine bioluminescence: the role of the in-water scalar irradiance, Appl. Optics 24, 1694 (1984).

8. S. Chandrasekhar, Radiative Transfer, Dover, New York, 1960, p. 304.

9. L.B. Barichello and M.T. Vilhena, A general approach to one-group one-dimensional transport equation, Kerntechnik 58, 182 (1993). 
10. A. Morel, Light and marine photosynthesis: a spectral model with geochemical and climatological implications, Prog. Oceanogr. 26, 263 (1991).

11. H. R. Gordon, A. Morel, Remote assessment of ocean color for interpretation of satellite visible imagery, a review, Lectures Notes on Coastal Estuarine Studies, vol. 4, Springer Verlag, 1983, p. 114.

12. A. Morel and L. Prieur, Analysis of variations in ocean color, Limnol. Oceanogr. 22, 709 (1977).

13. M. Dorigo, V. Maniezzo and A. Colorni, The ant Optimization: optimization by a colony of cooperating agents, EEE Trans. Syst. Man Cybernet.-Part B 26, 29 (1996).

14. E. Bonabeau, M. Dorigo and G. Theraulaz, Swarm Intelligence: From Natural to Artifical Optimizations, Oxford University Press, New York, 1999,.p. 309.

15. R.P. Souto, H.F. de Campos Velho, S. Stephany, A.J. Preto, C. F. Segatto, M.T. Vilhena, A Parallel Implementation of the LTSN Method for a Radiative Transfer Problem, The 15th Symposium on Computer Architecture and High Performance Computing (SBAC-PAD 2003), 10-12 November, São Paulo (SP), Brazil, ISBN 0-7695-2046-4, 116 (2003)

16. R.P. Souto, H.F. de Campos Velho, S. Stephany, E.S. Chaulhoub, Performance Analysis of Radiative Transfer Algorithms in a Parallel Environment, International Conference on Transport Theory (18-ICTT), 20-25 July, Rio de Janeiro, Brazil, 69 (2003).

17. P.K. Lamm, Inverse problems and ill-posedness. Inverse Problems in Engineering:Theory and Practice, ed. by N. Zabaras, K.A. Woodbury and M. Raynaud, ASME, pp. 1-10, Palm Coast, 1993.

18. A.N. Tikhonov and V.S. Arsenin, Solutions of Ill-Posed Problems, Winston and Sons, Washington, 1977.

19. R.P. Souto, S. Stephany, H.F. de Campos Velho, Reconstruction vertical profiles of absorption and scattering coefficients from multiespectral radiances, Mathematics and Computers in Simulation, 73, 255 (2006).

20. R.P. Souto, M. Kampel, F. Brandini, H.F. de Campos Velho, S. Stephany (2005): Comparative analysis of the use of "in situ" measurements and remote sensing ocean color data for Chlorophyll-A concentration, XII Brazilian Symposium on Remote Sensing (SBSR-2005), 16-21 Abril, Goiania (GO), Brazil, Proc. in CD-Rom: pp. 3679-3686 - in Portuguese.

21. R.P. Souto, V.C.F. Barbosa, H.F. de Campos Velho, S. Stephany (2007): Determining Chlorophill Concentration in Off-shore Sea Water from Multi-spectral Radiances by Using Second Devirtive Criterion and Ant Colony MetaHeuristic, Inverse Problems, Desing and Optimization Symposium IPDO-2007, April 16-18, Miami (FL), USA.

22. R.P. Souto, M. Kampel, H.F. de Campos Velho, S. Stephany (2007): Profile Retrieval of the Chlorophill-a Concentration, XII Latin-american Congress on Sea Sciences (COLACMAR-2007), April 15-19, Florianopolis (SC), Brazil - in Portuguese. 